\title{
Intraluminal urethral brachytherapy for recurrence of transitional cell carcinoma of urinary bladder in urethral stump
}

\author{
Bikramjit Chakrabarti, MD, DNB, Suman Ghorai, MD, Somapriya Basu Ray, MD, Sanjay Kumar Kar, MD \\ Institute of Post-Graduate Medical Education and Research, Kolkata, India
}

\begin{abstract}
We report a unique case of successfully performed intraluminal brachytherapy for low volume urethral mucosal recurrence of transitional cell carcinoma urinary bladder, initially treated by transurethral resection of bladder tumor, followed by radical cystectomy. Since the patient was unwilling to undergo any other operational interventions, intraluminal brachytherapy of urethra was attempted. Fluroscopy guided intraluminal HDR brachytherapy using Lumencath ${ }^{\circledR}$ catheter under local anesthesia, and remote afterloading system (Nucletron, an Elekta company, Elekta AB, Stockholm, Sweden) was performed. A fraction dose of $7 \mathrm{~Gy}$ in seven weekly fractions was prescribed at $0.5 \mathrm{~cm}$ from the single applicator. The result was promising in terms of local control and symptomatic relief. Therefore, intraluminal brachytherapy in low volume superficial local disease in urethra may play a potential role, and should be applied when repeated surgery is not feasible due to technical or medical reasons.

J Contemp Brachytherapy 2013; 5, 1: 42-44 DOI: $10.5114 /$ jcb.20l3.34451
\end{abstract}

Key words: bladder carcinoma, intraluminal brachytherapy, urethral recurrence.

\section{Purpose}

We report a unique case of successfully performed intraluminal brachytherapy for low volume urethral mucosal recurrence of transitional cell carcinoma urinary bladder, initially treated by transurethral resection of bladder tumor followed by radical cystectomy. A 40 year old man attended the oncologic out-patient department of our hospital with history of four months painless hematuria. Ultrasonography lower abdomen revealed a $4.3 \times 1.3 \mathrm{~cm}$ space occupying lesion in urinary bladder with bilateral mild hydronephrosis. The patient underwent trans-urethral resection of bladder tumor (TURBT). The polypoidal growth occupying left postero-lateral wall, and extending to anterior wall was resected out. Left ureteric orifice was not visualised. Postoperative histopathology reported low grade transitional cell carcinoma (TCC) with deep muscle involvement. Postoperative computerised tomographic scan (CT) revealed mild wall thickening of bladder at postero-lateral aspect with dilated collecting system of left kidney. Investigations revealed metastasis nowhere. However, post-operative cystoscopy showed multiple small to large bladder tumors. The largest one was $3 \mathrm{~cm}$. Radical cystectomy with nodal dissection and ileal conduit was done. Post-operative histopathology disclosed papillary transitional cell carcinoma, WHO grade II, infiltrating lamina propria, and involving detrusor, no lymphovascular invasion was seen, no invasion of prostate or seminal vesicles were present. Small tumors revealed papillary transitional cell carcinoma grade II. All lymph nodes indicated non-specific lymphatic hyperplasia. Biopsy taken from urethra indicated focal areas of hyperplastic thickening. No evidence of invasion was seen. Whole body bone scan was negative, and patient was put on follow up.

Patient was well until third month of follow up when he complained of episodes of urethra bleeding. Urethroscopy revealed presence of papillary growth near urethral stump, just proximal to membranous urethra up to a length of $3 \mathrm{~cm}$. Biopsy revealed low grade papillary transitional cell carcinoma. Computerised tomographic (CT) scan was within normal limits. Thus, a low volume superficial recurrence was diagnosed.

Since patient was unwilling to undergo any further operative interference, intraluminal brachytherapy of urethra was attempted. Fluroscopy guided intraluminal HDR brachytherapy using Lumencath ${ }^{\circledR}$ catheter under local anesthesia, and remote afterloading system (Nucletron, an Elekta company, Elekta AB, Stockholm, Sweden) was performed. A fraction dose of $7 \mathrm{~Gy}$ in seven weekly fractions was prescribed at $0.5 \mathrm{~cm}$ from the single applicator surface.

\section{Technique}

After proper antiseptic dressing and draping, $10 \mathrm{ml} 4 \%$ lignocine was instilled to the urethra and 6 French, $150 \mathrm{~cm}$ Lumencath ${ }^{\circledR}$ catheter was slowly inserted into the urethra 
through the recurrence up to the closed end of urethra (Fig. 1). C-arm based orthogonal imaging was done because of unavailability of planning DICOM based CT scan facility. Planning was done using PLATO brachytherapy planning system version $14.3 .7^{\circledR}$ (Nucletron, an Elekta company, Elekta AB, Stockholm, Sweden), using multiple points as reference points for dose prescription placed at $0.5 \mathrm{~cm}$ from the applicator with an active length of $5 \mathrm{~cm}$ (Fig. 2). A dose of 7 Gy was prescribed at these reference points, and a total dose of 49 Gy was given through 7 weekly fractions (EQD2 = 69,42 Gy). No organ was considered at risk radially within $2 \mathrm{~cm}$ of the growth.

Treatment was well tolerated by the patient. The only complaint was mild to moderate perineal pain during last three fractions of intraluminal brachytherapy, which was treated by oral non-steroidal anti-inflammatory drugs.

\section{Results}

The patient is clinically well (with no symptoms of bleeding per urethra, discharge or pain) since brachytherapy and repeated cystoscopy after three, six, nine months and one year of follow up proved complete regression of the growth in urethral stump. Random biopsy from the involved area revealed no malignancy. No palpable loco-re-

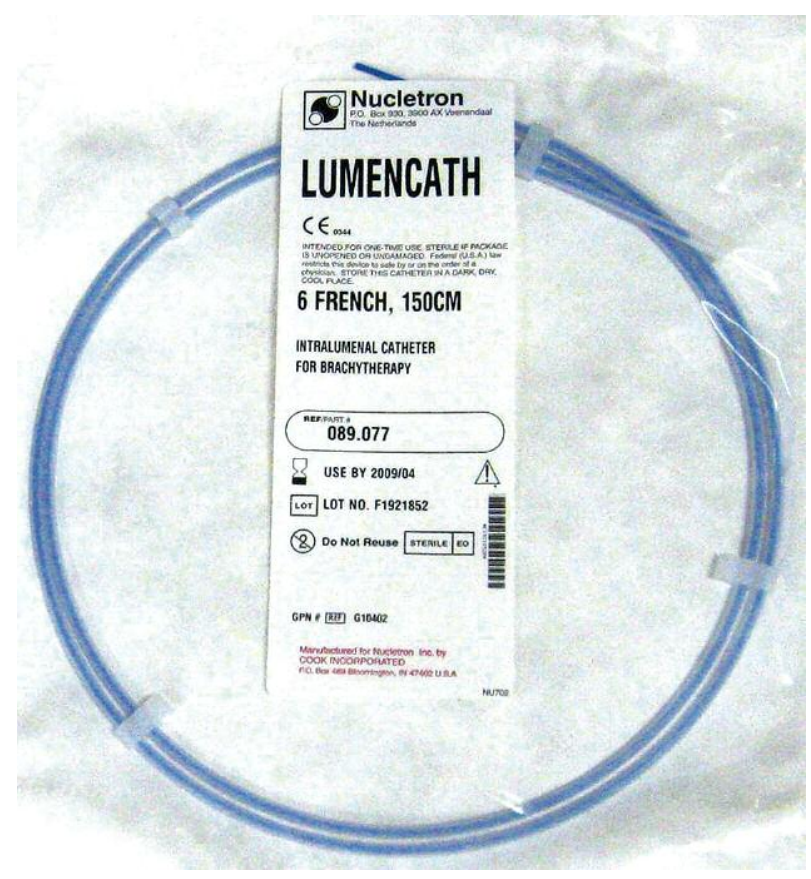

Fig. 1. Lumencath (Nucletron) intra-luminal catheter $(6 \mathrm{~F}$, $150 \mathrm{~cm}$ )
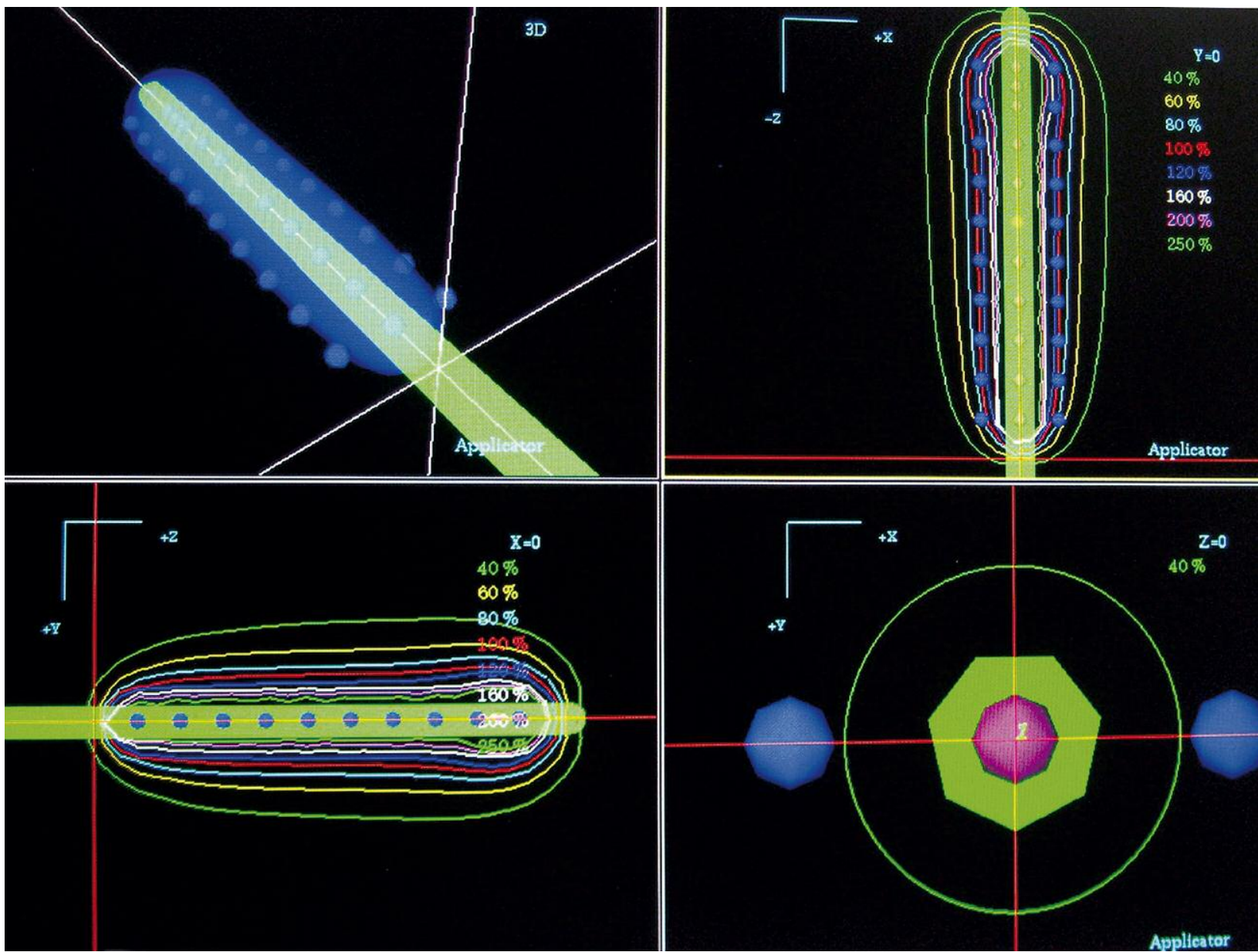

Fig. 2. Screenshot of the plan (PLATO version 14.3.7) 
gional nodes were detected on clinical examination. CT scan at one year was normal. The ileal conduit performed was regular.

\section{Discussion}

Urethra is considered as organ at risk during brachytherapy of pelvic organs like prostate [1] and vagina [2]. Urethral stricture is one of the most feared consequences after brachytherapy. Overall, $45.5 \%$ of stricture cases were of iatrogenic etiology (surgery, urethral interference and brachytherapy) [3]. The mean D2cc for urethra was $76( \pm 16)$ Gy after vaginal brachytherapy [2]. Data on cancers of the urethra are few and inconclusive [4]. Surgery, till date, is the curative treatment of carcinoma in urethra. Results for definitive radiotherapy in carcinoma involving urethra using either or both external beam radiation therapy and brachytherapy, have shown excellent cure rates in men and women. The primary advantage of radiotherapy is organ preservation. Advanced tumours cases needs additional chemotherapy or radiation. Although literature is limited to case reports because of the rarity of the disease, markedly improved results compared with older results of surgery with or without radiation permit consideration [5]. Superficial luminal recurrence of low grade transitional cell carcinoma of urinary bladder near urethral stump is uncommon, and its treatment is difficult due to repeated surgery in these cases that needs an expertise, and may add further morbidity to patient's condition. In our case, since patient was unwilling to undergo any repeated surgical interference, and as there was only a low volume superficial luminal recurrence near the urethral stump at an accessible site, intraluminal high dose rate brachytherapy was attempted with a prior declaration that brachytherapy would be postponed if at any instance failure surgery is suggested as a preference. Challenges we have faced were scarce radiobiologic data regarding efficacy of brachytherapy in transitional cell carcinoma, involving urethra, and unavailability of planning CT scan facility. Result was promising in terms of local control and symptomatic relief.

Therefore, intraluminal brachytherapy in low volume superficial local disease in urethra may play a potential role, and should be applied when repeated surgery is not feasible due to technical or medical reasons. Even in the era of threedimensional volume based planning, in a developing country with limited resources like India, brachytherapy may be attempted with 2D planning if the $3 \mathrm{D}$ planning is not available.

\section{References}

1. Earley JJ, Abdelbaky AM, Cunningham MJ et al. Correlation between prostate brachytherapy-related urethral stricture and periapical urethral dosimetry: A matched case-control study. Radiother Oncol 2012; 104: 187-191.

2. Dimopoulos JC, Schmid MP, Fidarova E et al. Treatment of locally advanced vaginal cancer with radiochemotherapy and magnetic resonance image-guided adaptive brachytherapy: dose-volume parameters and first clinical results. Int J Radiat Oncol Biol Phys 2012; 82: 1880-1888.

3. Lumen N, Hoebeke P, Willemsen P et al. Etiology of urethral stricture disease in the 21st century. J Urol 2009; 182: 983-987.
4. Troiano M, Corsa P, Raguso A et al. Radiation therapy in urinary cancer: state of the art and perspective. Radiol Med 2009; 114: 70-82. [Article in Italian]

5. Koontz BF, Lee WR. Carcinoma of the urethra: radiation oncology. Urol Clin North Am 2010; 37: 459-466. 